\title{
Sportverletzungen an Hüfte und Becken
}

\author{
Julia Heinzmann, Tim Pohlemann
}

\section{Zusammenfassung}

Sportverletzungen an Hüfte, Becken und Leiste betreffen überwiegend die Weichteile wie Muskeln, Sehnen, Bänder und Schleimbeutel. Das Spektrum umfasst Prellungen, Zerrungen, Rupturen, Tendinitiden und Bursitiden. Darüber hinaus stellen Leistenschmerzen ein häufiges Problem bei Sportlern dar. Aufgrund der Komplexizität der Anatomie von Hüfte, Becken und Leiste stellen die Weichteilverletzungen in diesem Bereich eines der schwierigsten Probleme in der Sportmedizin dar. Die Diagnosefindung erfordert neben einem umfassenden Wissen der differenzialdiagnostischen Möglichkeiten häufig eine interdisziplinäre Zusammenarbeit von Unfallchirurgen, Orthopäden, Allgemeinchirurgen, Gynäkologen und Neurologen. Frakturen treten in der Regel seltener auf. Meist handelt es sich dann um unkomplizierte Abriss- oder Stressfrakturen mit überwiegend konservativer Therapie. Schwere Frakturen von Becken, Hüfte und Azetabulum entstehen entweder durch Rasanztraumen bei Sportarten wie Skifahren, Motorsport und Reiten oder durch Sturz aus großer Höhe wie beim Fallschirmspringen, Bergsteigen etc. Die Behandlung dieser Frakturen richtet sich nach den Kriterien der Beckenchirurgie.

\section{Sport-Related Injuries of the Hips and Pelvis}

Sport-related injuries to the hips, pelvis and groin mostly involve soft tissues such as muscle, tendon, ligament and bursa. The spectrum encompasses contusions, sprains, ruptures, inflammations of the tendons or bursa. In addition, pain in the groin is a frequent problem for athletes and players. On account of the anatomic complexity of the hips, pelvis and groin, soft tissue injuries in this region constitute one of the most difficult problems in sports medicine. Besides a comprehensive knowledge of the differential diagnostic possibilities, often interdisciplinary cooperation between accident surgeons, orthopaedic surgeons, general surgeons, gynaecologists and neurologists is required to make a diagnosis. Most cases, however, involve uncomplicated avulsion or stress fractures that are amenable to conservative treatment. Severe fractures of the pelvis, hips and acetabulum occur due to high-speed trauma in activities like skiing, motor sport and riding or to falls from a great height as in parachuting or mountain climbing. Treatment of these fractures follows the criteria for pelvis surgery.

\section{Knöcherne Läsionen}

Becken

Bei Sportlern treten überwiegend TypA-Verletzungen auf, die den knöchernen Beckenring nicht unterbrechen. Dazu gehören Schambeinastfrakturen, Iliumfrakturen oder quere sakrale bzw. kokzygeale Frakturen. Höhergradige Beckenringfrakturen entstehen durch Rasanztraumen bei Sportarten mit hoher Geschwindigkeit wie Skifahren und Motorsport sowie durch Sturz aus großer Höhe beim Fallschirmspringen oder Bergsteigen. Darüber hinaus führen Reitunfälle in Abhängigkeit von der Kollision des Reiters mit dem Pferd zu lateralen Kompressions- oder „Open-Book“-Ver-

OP-JOURNAL 2008; 24: 84-94

(c) Georg Thieme Verlag KG Stuttgart • New York DOI 10.1055/s-2008-1038823 letzungen des Beckens. Die Behandlung dieser Frakturen richtet sich nach den Kriterien der Beckenchirurgie.

Frakturen können durch direktes oder indirektes Trauma entstehen. Bei Letzteren unterscheidet man wiederum die Abrissfraktur von der Stressfraktur, welche als Folge einer chronischen Überlastung auftritt.

\section{Abrissfraktur}

Im Bereich des Beckens finden sich diese Frakturen typischerweise bei jugendlichen Sportlern, jedoch auch bei Adoleszenten als Apophysenabrisse (Avulsionsfraktur). Bei Apophysen handelt es sich um sekundäre Ossifikationszentren, welche im Gegensatz zu den Epiphysen keinen Einfluss auf das Längenwachstum haben. Der Entstehungsmechanismus eines knöchernen bzw. Apophysenausrisses ist eine plötzliche und uner- wartete Belastung eines bereits kontrahierten Muskels. Diese tritt in der Beschleunigungsphase oder beim abrupten Abbremsen einer Bewegung auf. Die Ursache ist darin zu sehen, dass Bänder und Sehnen bei Jugendlichen und jungen Erwachsenen einer größeren Kraft standhalten können als die knöchernen Bestandteile des Beckens.

\section{Abrissfraktur der Spina iliaca anterior superior (Abb.1)}

Zwischen dem 13. und 15. Lebensjahr werden die Knochenkerne radiologisch sichtbar. Der Fugenschluss liegt zwischen dem 21. und 25. Lebensjahr.

Ursache: plötzliche Kontraktion des M. sartorius sowie Hyperextension im Rumpf (Bogenspannung).

Disziplin: Lauf, Sprung, Wurfdisziplinen. 


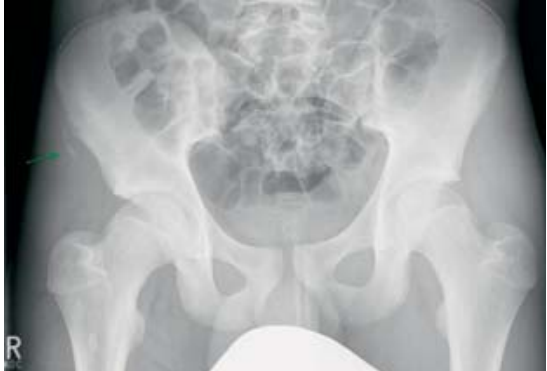

Abb. 1 Abrissfraktur der Spina iliaca anterior superior bei einem 16-jährigen Jungen nach Schuss beim Fußball.

Mit freundlicher Genehmigung der Klinik für Diagnostische Radiologie, Universitätsklinikum des Saarlandes (Prof. Dr. A. Bücker).

Symptome: Unterbrechung der durchgeführten Bewegung. Schmerzhafte Einschränkung der passiven Hüftextension und aktiven Hüftbeugung.

Diagnostik: Sonografie, Röntgen: Beckenübersicht, ggf. Ala-Aufnahme (Patient wird um 30 Grad zur verletzten Seite gekippt).

Therapie: konservativ: Teilbelastung der betroffenen Seite bis zur Schmerzfreiheit. Anschließend muskelaufbauende Übungsbehandlung.

Ausnahme: operative Therapie bei Dislokation des Fragmentes $>2 \mathrm{~cm}$ oder bei Hochleistungssportlern, wenn mit einem Funktionsdefizit bzw. einem Verlust der Muskelkraft zu rechnen ist. Darüber hinaus bei funktionell störender Kallusbildung. Das Fragment wird in dem Fall mit einer Zugschraubenosteosynthese oder einer Cerclage refixiert.

\section{Abrissfraktur der Spina iliaca anterior inferior (Abb. 2)}

Die Knochenkerne erscheinen ebenfalls zwischen dem 13. und 15. Lebensjahr, fusionieren jedoch bereits zwischen dem 16. und 18. Lebensjahr.

Ursache: plötzliche Kontraktion des M. rectus femoris.

Disziplin: typische Sprintverletzung (Start), Ballspiele (Fußball, Hockey).

Symptom: Einschränkung der aktiven Kniestreckung.

Diagnostik: klinisch: schmerzhafte Extension des Kniegelenkes gegen Widerstand. Sonografie, Röntgen: Beckenübersicht.

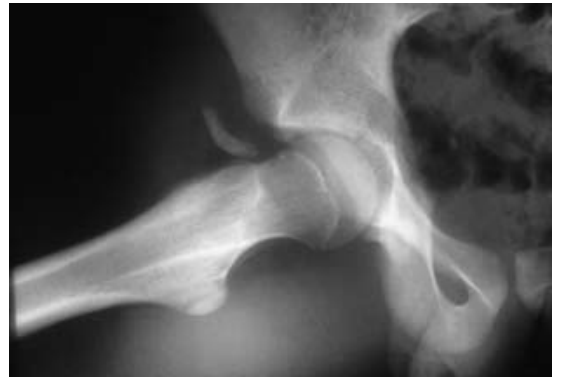

Abb. 2 Abrissfraktur der Spina iliaca anterior inferior bei einem 16-jährigen Jungen.

Mit freundlicher Genehmigung der Klinik für Unfall-, Hand- und Wiederherstellungschirurgie, Universitätsklinikum des Saarlandes.

Therapie: entspricht der Abrissfraktur der Spina iliaca anterior superior.

Abrissfraktur des Tuber ischiadicum

(Abb. 3)

Die Knochenkerne erscheinen zwischen dem 14. und 16. Lebensjahr und fusionieren zwischen dem 18. und 25. Lebensjahr.

Ursache: plötzliche Kontraktion der ischiokruralen Muskulatur oder des M. adductor magnus.

Disziplin: Sprint, Sprung (Hoch- und Weitsprung), Hürdenlauf, Längsspagat beim Turnen, Gewichtheben.

Symptome: Schmerzen beim Gehen in der Phase der Hüftstreckung sowie beim Sitzen.

Diagnostik: klinisch: Schmerzen bei Hüftbeugung mit gestrecktem Knie und Rotation, Schmerzen bei passiver Dehnung der Hüftstrecker und Adduktoren.

Sonografie, Röntgen: Beckenübersicht.

Therapie: konservativ: Teilbelastung der betroffenen Seite bis zur Schmerzfreiheit. Anschließend aufbauende Übungsbehandlung.

Ausnahme: operative Therapie bei starker Dislokation des Fragmentes, großer Kallusbildung mit Schmerzen sowie Einschränkungen beim Sitzen.

Cave: Diese Frakturen können mit Läsionen des $\mathrm{N}$. ischiadicus einhergehen. Posttraumatische Kallusbildungen können Sensibilitätsstörungen hervorrufen. Nicht selten wird die Kallusbildung als Osteosarkom fehlgedeutet!
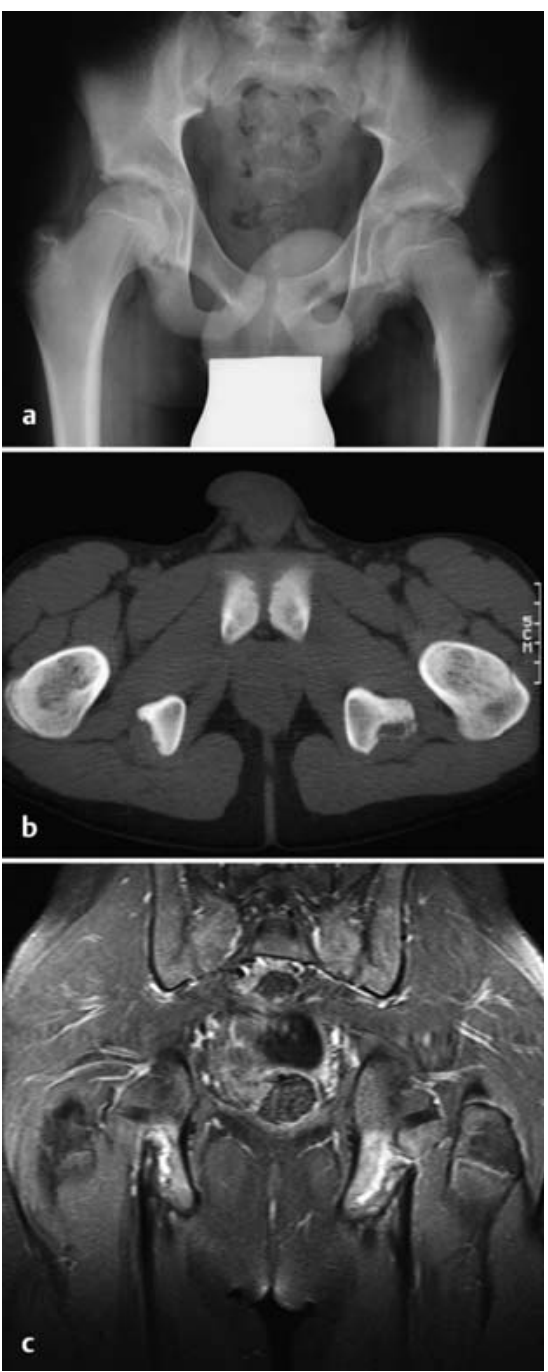

Abb. 3 a bis c Abrissfraktur des Tuber ischiadicum bei einem 16-jährigen Fußballspieler nach intensivem Sprinttraining. a Beckenübersicht. b Becken-CT. c Becken-MRT: Im Gegensatz zum Nativröntgen und CT, welche lediglich die Avulsionsfraktur des linken Sitzbeines zeigen, ist hier die Signalanreicherung in beiden Sitzbeinen ein Zeichen rezidivierender Mikrotraumata.

Mit freundlicher Genehmigung der Klinik für Diagnostische Radiologie, Universitätsklinikum des Saarlandes (Prof. Dr. A. Bücker).

\section{Abrissfraktur am Beckenkamm}

Die Knochenkerne erscheinen zwischen dem 13. und 15. Lebensjahr und fusionieren mit dem 25. Lebensjahr. An der Christa iliaca setzen ventral die Bauchwandmuskeln (M. obliquus abdominis externus, M. obliquus abdominis internus und $\mathrm{M}$. transversus abdominis) an. Dorsal ist sie der Ursprung der Glutealmuskulatur.

Disziplin: beim Läufer als Abrisse, beim Football durch direktes Trauma. 
Symptome: schmerzhafte Kontraktion der Bauchwandmuskulatur, vor allem mit Rotationsbewegungen, Anspannung der Glutealmuskulatur.

Diagnostik: Röntgen: Beckenübersicht.

Therapie: entspricht der Abrissfraktur der Spina iliaca anterior superior.

\section{Abrissfraktur des Schambeinastes}

Ursache: Abriss des M. adductor magnus (ventral) oder M. adductor longus bzw. M. gracilis (symphysennah). Dorsal setzt die ischiokrurale Muskulatur an.

Disziplin: Skifahren, Turnen, Eiskunstlauf.

Symptome: medialseitige Oberschenkelschmerzen, die in das Knie ausstrahlen.

Diagnostik: klinisch: Die Abduktion der Beine mit Hyperextension der Hüfte ist schmerzhaft. Längsspagat ist bei Verletzung der ischiokruralen Muskulatur nicht durchführbar. Röntgen: Beckenübersicht.

Therapie: konservativ.

\section{Stressfrakturen (Ermüdungsbruch)}

Bei der Stressfraktur handelt es sich um eine partielle oder vollständige Fraktur des Knochens als Folge einer wiederholten Mikrotraumatisierung nach Fehloder Überlastung bei gleichzeitigen Reparationsvorgängen. Es existieren zwei Theorien über die Ursache der Stressfrakturen. Die Ermüdungstheorie besagt, dass die Muskulatur nach wiederholten und andauernden Beanspruchungen ihre Belastungsgrenze überschreitet und dann nicht mehr in der Lage ist das Skelett zu unterstützen, z. B. beim Aufprall, wenn der Fuß den Boden berührt. Die Belastung wird dann direkt auf das Skelett übertragen. Nach Überschreiten der Toleranz tritt dann eine Fraktur auf

Die Überlastungstheorie beruht auf der Tatsache, dass Muskelgruppen sich derart kontrahieren, dass die Knochen, an denen sie ansetzen, biegen. Nach wiederholten Kontraktionen wird die Knochenstärke überschritten und er bricht. Nahezu jeder Knochen im Körper kann eine Stressfraktur erleiden. Sie treten jedoch nicht ein zweites Mal an der gleichen Stelle auf. Insgesamt treten diese Verletzungen bei gesunden Personen ab dem 7. Lebensjahr auf. Die Inzidenz ist bei jungen Sportlern und Frauen erhöht. Ein Risikofaktor besteht in der sog. „Sportlerinnen-Triade“. Dieses Syndrom fasst drei Beschwerden zusammen, die in wechselseitiger Beziehung stehen: Ess-Störungen, Amenorrhöe und Osteoporose. Dieses Syndrom ist besonders unter Ausdauerläuferinnen sowie beim Kunstturnen, Eiskunstlaufen und Ballett weitverbreitet.

Problematisch ist die Diagnose der Stressfraktur, da sie in den ersten 4-6 Wochen auf konventionellen Röntgenbildern nicht oder nur unzureichend sichtbar sind. Die Kernspintomografie oder 3-Phasen-Skelettszintigrafie ist bei entsprechendem Verdacht das diagnostische Mittel der Wahl. 6-8\% aller Stressfrakturen betreffen das Femur. Handelt es sich um einen Ermüdungsbruch des Schenkelhalses, ist die frühzeitige Diagnose von entscheidender Bedeutung, da häufig eine operative Versorgung erforderlich ist, um eine vollständige Heilung unter funktioneller Nachbehandlung zu gewährleisten und eine Dislokation mit in der Folge auftretender Femurkopfnekrose zu vermeiden.

Cave: Wenn Sportler, insbesondere Langstreckenläufer über Leistenschmerzen klagen, muss eine Stressfraktur des Schenkelhalses ausgeschlossen werden. Differenzialdiagnostisch muss an einen Ermüdungsbruch der vorderen Schambeinäste gedacht werden!

Symptome: in 50\% der Fälle schleichender Beginn, 50\% akut ohne offensichtliche Verletzung. Die Schmerzen sind zunächst aktivitätsabhängig, treten im weiteren Verlauf aber auch nachts auf.

Diagnostik: Die konventionelle Röntgenaufnahme hat eine geringe Sensitivität bei hoher Spezifität. Sie zeigt neue periostale Knochenformationen, einen Sklerosebereich und Kallus oder eine Frakturlinie. Diese Zeichen treten jedoch erst 4 Wochen nach Symptombeginn auf. Die 3-Phasen-Knochenszintigrafie hat dagegen eine Sensitivität von 100\%. Knöcherne Veränderungen können bereits 48-72 h nach Beginn der Symptome festgestellt werden. Der größte Nachteil ist die fehlende Spezifität, da auch andere Läsionen wie z.B. Tumoren oder Infekte zu einer lokalen Signalanreicherung führen können. Genauso früh können die knöchernen Veränderungen, wie z.B. eine lokale Ödembil- dung, im MRT diagnostiziert werden bei deutlich besserer Spezifität und fehlender Strahlenbelastung der meist noch jungen Patienten.

Therapie: meist konservativ: Schonung und ggf. Entlastung an Unterarmgehstützen für 4-8 Wochen. Rückkehr zum Sport erst nach vollständiger Schmerzfreiheit. Kondition durch Schwimmen oder Radfahren aufrechterhalten.

Eine Indikation zur operativen Therapie besteht bei Lokalisation der Stressfraktur im Schenkelhals. Hier kann z. B. eine Verschraubung als relativ kleiner operativer Eingriff mit anschließender funktioneller Nachbehandlung und rascher Aufbelastung sinnvoll sein.

\section{Osteitis pubis}

Dabei handelt es sich um eine schmerzhafte Entzündung von Symphyse, Schambeinknochen und angrenzender Weichteilstrukturen (Adduktoren, Bauchmuskulatur, Faszien).

Ursache: rezidivierende Mikrotraumatisierung durch Fehl- oder Überlastung durch dauerhaft hohe antagonistisch wirkende Muskelkräfte (Bauchmuskulatur versus Adduktoren) auf die sehnigen und periostalen Ansätze an Symphyse und Schambeinästen.

Disziplin: Sportarten mit Sprint- und Schusselementen sowie schnellen Richtungswechseln (Fußball, Basketball, Laufsportarten). Die Inzidenz bei Sportlern liegt bei $0,5-7 \%$.

Symptome: Schmerzen beim Gehen, Treppensteigen und Einbeinstand. Lokal auf Symphyse oder Schambeinäste begrenzt, aber auch ausstrahlend in Leiste, Hüfte oder Perinealregion.

Diagnostik: klinische Untersuchung: Druckschmerz über Symphyse bzw. Schambeinästen. Positiver „SymphysenKlaff-Test" (Schmerzprovokation über Adduktorenansätzen auf Druck sowie bei isometrischer Anspannung gegen Widerstand).

Konventionelle Beckenübersichtsaufnahme: Erosionen über der Symphyse, vermehrte subchondrale Sklerose, zystische Veränderungen. Häufig Symphysengelenkspaltweite $>10 \mathrm{~mm}$. 
„Flamingoaufnahme“ (a.-p. Aufnahme der Symphyse im alternierenden Einbeinstand rechts- und linksseitig): Vertikale Verschiebung der Symphyse $>2 \mathrm{~mm}$ auf der belasteten Seite.

Drei-Phasen-Skelettszintigrafie: Abgrenzung von der Osteomyelitis. Anreicherung in der Demineralisations- und Spätphase. Bei einer Osteomyelitis sind alle 3 Phasen positiv.

MRT: subchondrales Knochenödem, Flüssigkeit im Symphysenspalt (akut), subchondrale Sklerose und knöcherne Irregularitäten und Osteophyten (chronisch).

Des Weiteren sollten bei diesen Patienten auch immer die Sakroiliakalgelenke in die klinische und bildgebende Diagnostik mit einbezogen werden. In einer Studie von Major et al. (1997) konnte gezeigt werden, dass bei Sportlern, die über Schmerzen im Bereich der Symphyse, Leiste oder ischialgieforme Beschwerden sowie die Kombination davon klagten, eine bildmorphologisch nachgewiesene Osteitis pubis häufig assoziiert war mit Veränderungen der SI-Gelenke. Diese reichten von degenerativen Veränderungen bis hin zur sakralen Stressfraktur. Eine mögliche Erklärung wäre, dass die zu hohe Be- oder Überlastung über die knöcherne Ringstruktur des Beckens fortgeleitet wird und zu sekundären Veränderungen im dorsalen Beckenring führt.

Therapie: zunächst immer konservativ: physikalische Therapie, Antiphlogistika, Kryotherapie, Physiotherapie mit Kräftigung von Rumpf- und Beckenmuskulatur, Dehnung der Adduktoren, Sportpause. Als weitere diagnostische und therapeutische Maßnahme kann die Infiltration des Symphysengelenkspaltes mit einer Kombination aus Lokalanästhetikum und Kortikosteroid unter Bildwandlerkontrolle indiziert sein.

Operative Therapie: Kürettage oder Arthrodese: als Ultima Ratio bei chronisch schmerzhafter und therapieresistenter Osteitis pubis nach monatelanger konservativer Therapie mit ausbleibender Beschwerdebesserung.

\section{Hüftgelenk}

\section{Luxation des Hüftgelenkes}

Zur Luxation des Hüftgelenkes kommt es nach Hochrasanz- bzw. Anpralltraumata mit entsprechender Krafteinwirkung wie sie beim Motorsport („dash board injury“), Reitsport oder American Football auftreten. Am häufigsten ist die Luxation nach dorsal in Kombination mit einer Azetabulumfraktur.

Symptome: Der Patient hat starke Schmerzen, das Bein ist verkürzt und innenrotiert.

Diagnostik: klinisch, Röntgen: Beckenübersicht.

Therapie: sofortige Klinikeinweisung und Reposition des Gelenkes unter Analgesie, bei Luxation ohne weitere Fraktur kann eine Kurznarkose erforderlich sein. Anschließend Durchführung eines Becken-CT zum Ausschluss eines osteochondralen Flake bzw. bei zusätzlicher Azetabulumfraktur zur Planung der sekundär operativen Versorgung.

Die Verletzung kann im Verlauf von Monaten bis Jahren eine Hüftkopfnekrose zur Folge haben, die zu einer andauernden Behinderung führt und schließlich einen endoprothetischen Gelenkersatz erforderlich macht.

\section{Schenkelhalsfraktur}

Dabei handelt es sich um häufige Verletzungen des älteren Menschen. Sie treten jedoch auch bei jüngeren durch direkten Sturz auf die Hüfte wie z. B. beim Schlittschuhlaufen, Skifahren etc. auf. (s. auch Stressfraktur).

Symptome: Das betreffende Bein ist typischerweise verkürzt und außenrotiert.

Diagnostik: klinisch, konventionelle Röntgenaufnahme der Hüfte in 2 Ebenen.

Therapie: operativ durch Schrauben, DHS etc., Ziel ist die kopferhaltende Osteosynthese.

Intraartikuläre Veränderungen des Hüftgelenkes

Die im Folgenden genannten Veränderungen des Hüftgelenkes sind weder spezifisch für die Patientengruppe der Sportler noch für bestimmte Sportdis- ziplinen. Da Schmerzen in der Leiste jedoch häufig vom Hüftgelenk fortgeleitet werden, müssen diese in die Diagnostik mit einbezogen werden. Von entscheidender Bedeutung ist zunächst die Abgrenzung einer intraartikulären Läsion von einer periartikulären Problematik (im Folgenden unter „II. Weichteilverletzungen“ beschriebenen Schädigungen). Dazu gehören degenerative Veränderungen, die unter sportlicher Belastung häufig früher symptomatisch werden, freie Gelenkkörper, Osteochondrosis dissecans, Labrumläsionen, Ruptur des Ligamentum capitis femoris, ein femoroazetabuläres Impingement oder eine Psoastendinitis.

Symptome: Schmerzen zunächst unter Belastung in Hüftgelenk und Leiste, später auch in Ruhe, Bewegungseinschränkungen, Blockierungen des Gelenkes.

Diagnostik: klinisch: Druckschmerz im Bereich der Leiste oder des Trochanter major, eingeschränktes Bewegungsausmaß (Neutral-Null-Methode), Schmerzen bei Rotation in 90 Grad Hüftflexion sowie in Extension, Gangbild, Einbeinstand.

Bildgebung: konventionelles Röntgen: Hüfte a.-p. und Lauenstein zur Beurteilung degenerativer Veränderungen, freier Gelenkkörper, knöcherner Impingementzeichen.

MRT zur Beurteilung von Knorpelschäden, Knochenzysten am Pfannenerker oder Kopf-Hals-Übergang, Labrumläsionen, Ergussbildung.

Arthro-MRT bei Knorpelschäden, Labrumläsionen, Ruptur des Lig. capitis femoris, Chondrome.

Positiver Lokalanästhetikum-Test: Zur endgültigen Differenzierung einer intraartikulären von einer periartikulären Läsion dient die probatorische intraartikuläre Infiltration des Hüftgelenkes mit einem Lokalanästhetikum unter Bildwandlerkontrolle. Ist der Patient danach vorübergehend beschwerdegebessert oder schmerzfrei, handelt es sich um eine intraartikuläre Problematik.

Therapie: Bestätigt sich durch die angegebene Diagnostik eine der o.g. intraartikulären Veränderungen des Hüftgelenkes besteht prinzipiell die Indikation zur Hüftgelenksarthroskopie. Ausnahme: weitfortgeschrittene Koxarthrose. 


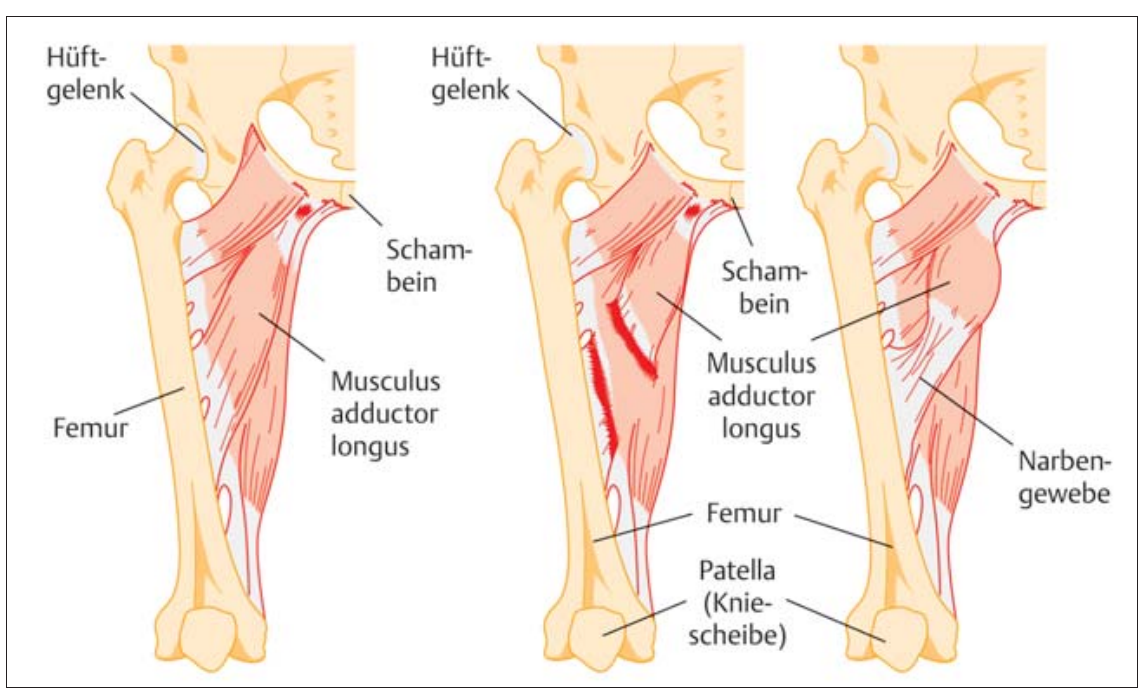

Abb.4 Im Bereich der Adduktoren treten häufig Verletzungen bis hin zu Rissen auf. Häufige Lokalisation ist der Muskelursprung am Os pubis (links). Riss im Muskelansatz am Oberschenkelknochen (Mitte). Heilung mit Narbengewebe (rechts).

Arthroskopisch durchführbar sind: Entfernung freier Gelenkkörper, Refixation eines osteochondralen Flake, Mikrofrakturierung, Knorpeldébridement, Synovektomie, Kopf-Hals-Taillierung bei Cam-Impingement (femorale Form des femoroazetabulären Impingements) oder eine Tenotomie der Psoassehne nach erfolgloser konservativer Behandlung.

\section{Hüftbeschwerden bei Kindern}

Schmerzen in Knie- und Hüftgelenken sowie ein Schonhinken bei Kindern und Jugendlichen müssen abgeklärt werden. Schmerzen, die vom Kind im Kniegelenk angegeben werden, haben ihren Ursprung häufig in einer Hüftgelenkerkrankung. Wichtig ist daher der Ausschluss bzw. das Erkennen der folgenden Erkrankungen: M. Perthes, Epiphysiolysis capitis femoris, Coxitis fugax, Synovialitis.

\section{Weichteilverletzungen}

Sportverletzungen an Hüfte und Becken betreffen überwiegend die Weichteile, d.h. Muskeln, Sehnen, Bänder und Schleimbeutel. Diese entstehen einerseits durch akute Überdehnung (inadäquate Bewegungsmomente), andererseits durch chronische Überlastung oder selten durch ein direktes Trauma. Die Diagnostik beinhaltet vor allem die Anamnese und klinische Untersuchung mit Inspektion, Palpation und funktioneller Untersuchung. Sie reicht oft aus, um eine korrekte Diagnose zu stellen. Dabei ist die Kenntnis der am Becken ansetzenden Muskeln und deren Zugrichtung die Grundlage, um Bewegungseinschränkungen sicher klassifizieren zu können. Eine bildgebende Diagnostik ist nur in seltenen Fällen und dann meist zum Ausschluss einer Abriss- bzw. Stressfraktur erforderlich.

\section{Verletzungen der Muskel-/Sehneneinheit}

Bei muskulären Dysbalancen (phasische versus tonische Muskulatur, Agonisten versus Antagonisten) kommt es zur Verschiebung des Gleichgewichtes der Gegenspieler und zur zunehmenden Verletzungsbereitschaft des Muskels. Für die Becken-Hüft-Region ist die Tendopathie die häufigste Verletzungsform. Differenziert werden Verletzungen am Ursprung oder Ansatz (Insertionstendopathie), der Sehne selbst oder am Muskel-Sehnen-Übergang. Die Ursache ist eine chronische Überbelastung mit stereotypen Bewegungsabläufen. Eine plötzliche Mehrbeanspruchung führt dann zur Verletzung. Bei langsamer Degeneration reicht meist eine Alltagsbelastung zum Riss einer Sehne. Die Therapie ist fast immer konservativ.

Wichtig ist die Prävention: Aufwärmund Auslauftraining, Kraft-, Koordinations- und Dehnungsübungen in jedem Training!

\section{Adduktorenverletzung}

Von allen Adduktoren (M. adductor brevis, M. adductor magnus, M. adductor longus, M. gracilis und untere Fasern des M. gluteus maximus) wird der M. ad-

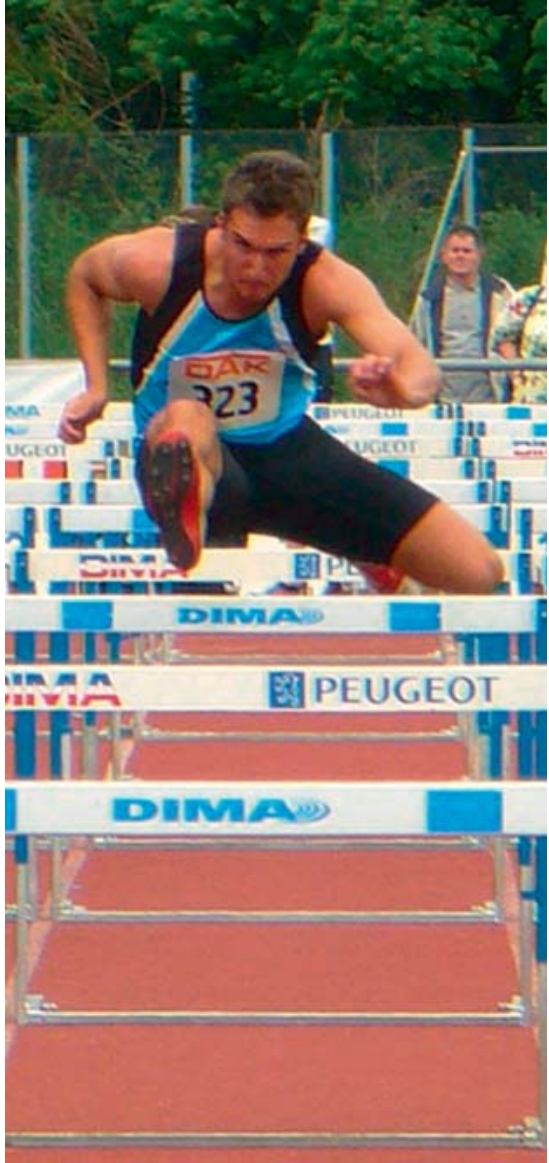

Abb. 5 Eine plötzliche Anspannung der Adduktoren tritt z. B. beim Hürdenlauf auf.

ductor longus am häufigsten bei Sportlern verletzt. Die Sehne entspringt am Schambein und setzt an der Femurmitte dorsal an. Die Adduktorenmuskulatur kann vollständig oder teilweise rupturieren. Vollständige Rupturen treten normalerweise am femurnahen Muskelansatz auf, Partialrupturen häufiger am Muskel-Sehnen-Übergang (Abb. 4).

Ursache: kräftige Kontraktion nach vorheriger Überlastung, Seitwärtstritte beim Fußball, hartes Laufbahntraining.

Disziplin: Fußball, Schlittschuhlaufen, Hürdenlauf, Hochsprung, Gewichtheben, Reiten, Turnen (Abb.5).

Symptome: Schmerzen an der Oberschenkelinnenseite, häufig ausstrahlend in die Leiste. Bei Rupturen plötzlicher, stechender Schmerz in der Leistenregion. Schwellung und Hämatomverfärbung bei lokaler Einblutung.

Diagnostik: lokaler Druckschmerz im Bereich des Muskelursprungs am Schambein. Schmerzauslösung durch Zusam- 
mendrücken der Beine gegen Widerstand. Der Sportler kann häufig nicht laufen, aber Rad fahren.

Bei Ruptur: Unfähigkeit der Muskelanspannung. Schwellung und Hämatomverfärbung, die häufig jedoch erst Tage nach der Verletzung auftreten. Tastbare Delle im Bereich der höchsten Druckempfindlichkeit. Sonografie. MRT (Abb. 6).

Therapie: konservativ: initial Kryotherapie (ersten $48 \mathrm{~h}$ ), dann lokale Wärmebehandlung. Antiphlogistika. Alle schmerzhaften Aktivitäten vermeiden. Grundkondition durch Radfahren oder Kraulschwimmen aufrechterhalten, jedoch nur bei Schmerzfreiheit. Ggf. lokale Steroidinjektion mit nachfolgender 1 2-wöchiger Schonung. Querfriktionsmassage. Bei sofortiger Behandlung heilt die Verletzung in aller Regel in 1-2 Wochen aus, vorausgesetzt der Sportler nimmt Training und Wettkampf erst nach vollständiger Schmerzfreiheit wieder auf. Ansonsten besteht das Risiko einer Chronifizierung.

Bei Ruptur: sofortige Kühlung, Kompression, Hochlagerung und Bandage. Entlastung des betroffenen Beines an Unterarmgehstützen. Im Verlauf Übungsbehandlung zur Aufrechterhaltung des Bewegungsumfanges, dann Radfahren, Schwimmen, leichtes Joggen mit zunehmendem Konditionstraining. Eine Teilruptur heilt unter Narbenbildung nach der akuten Phase aus.

Operation: Einkerbung der Sehnen und/ oder lokale Entfernung von geschädigtem Sehnengewebe als Ultima Ratio bei mehrmonatiger therapieresistenter konservativer Behandlung. Naht bei vollständiger Ruptur.

\section{Verletzung des M. iliopsoas}

Der Muskel entspringt an den Lendenwirbeln und an der Innenseite des Os ileum und setzt am Trochanter minor an. Er ist der stärkste Hüftbeuger. Dorsal der Sehne liegt die Bursa iliopectinea, welche die Sehne halbkreisförmig umgibt. Diese kann isoliert oder mit der Sehne entzündet sein. Eine Be- bzw. Überlastung betrifft überwiegend die Ansatzstelle (Abb. 7).

Ursache: Überlastung des Muskels, ggf. mit Bursitis. Verkürzung durch unausgewogenes Bauchmuskeltraining, die zu statischen und funktionellen Verän-

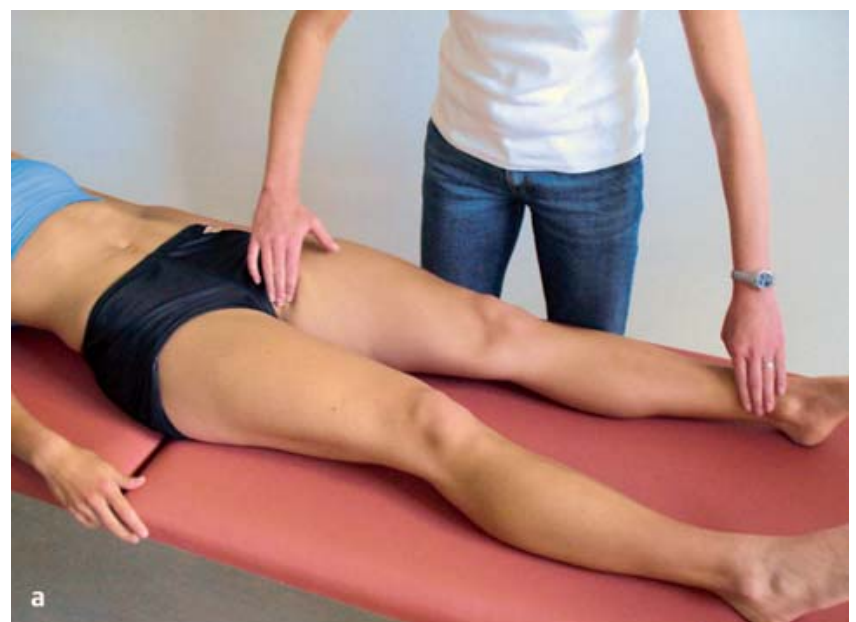

Abb. 6 a und $b$ Klinische Untersuchung: a Druckschmerz am Ursprung der Muskulatur am Schambein, b Schmerzauslösung durch Zusammendrücken der Beine gegen Widerstand.
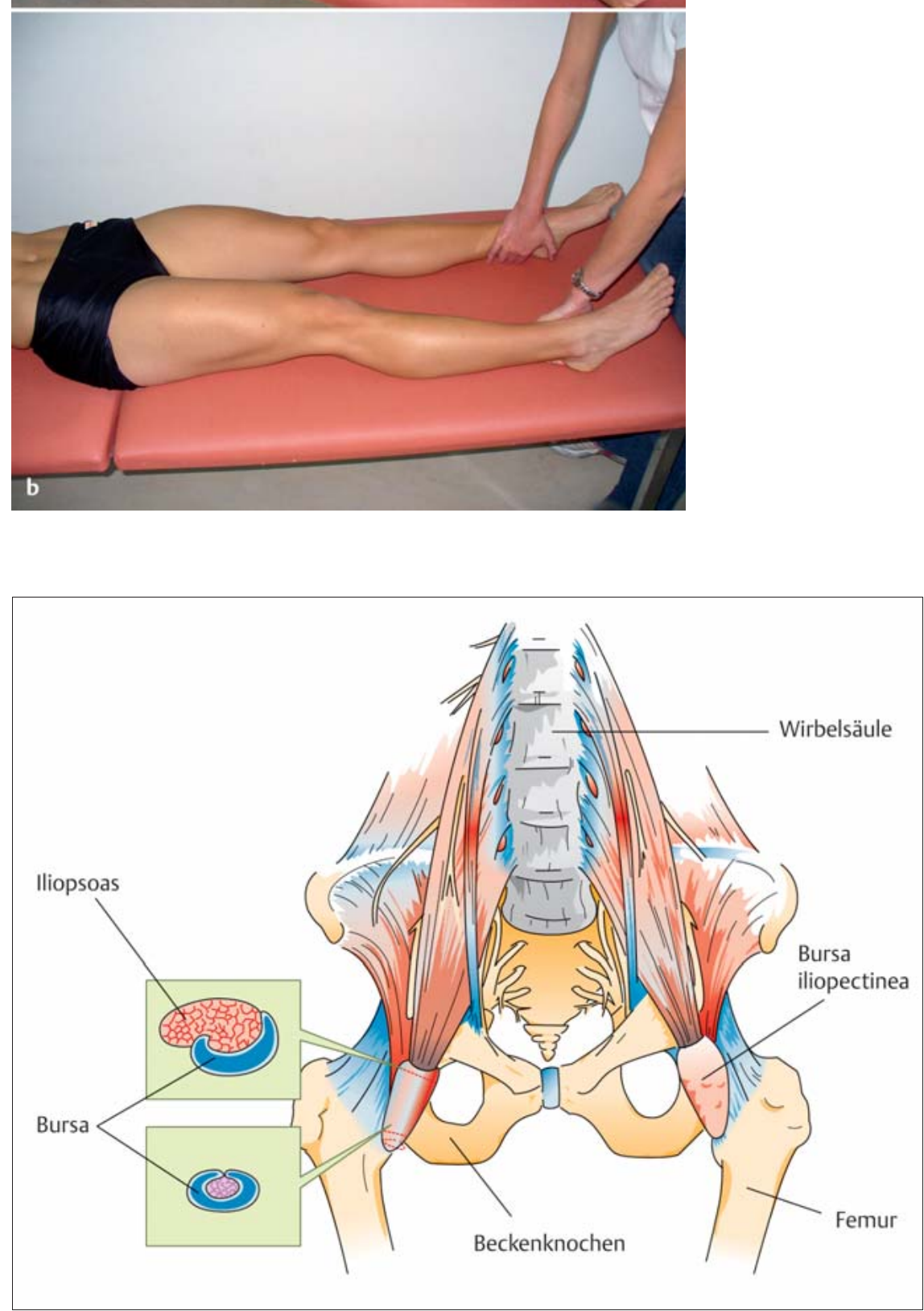

Abb. 7 Die Muskel-Sehnen-Einheit des M. iliopsoas und die Bursa iliopectinea. 

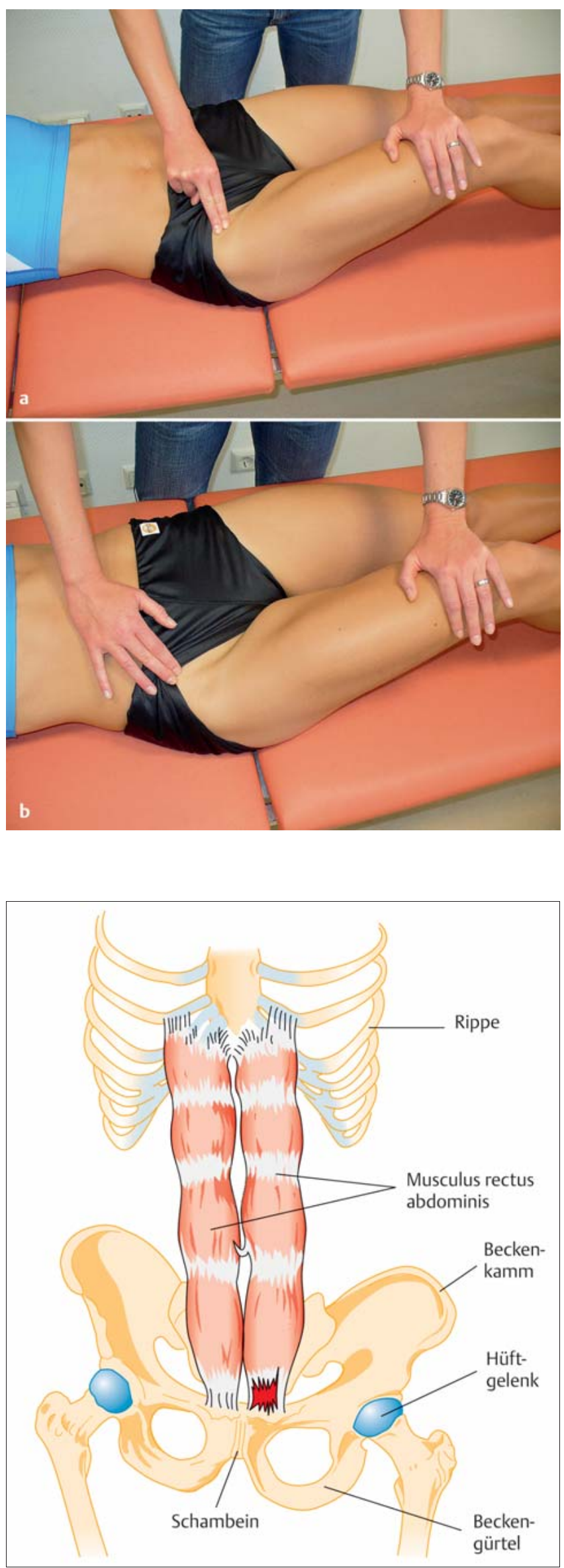

Abb. $8 \mathrm{a}$ und $\mathrm{b}$ Klinische Untersuchung: Schmerzauslösung bei Beugung des Hüftgelenkes gegen Widerstand.

Abb. 9 Riss am Ansatz des $M$. rectus abdominis am Schambein. derung der Lenden-Becken-Hüftregion führt. Rupturen treten meist am Sehnenansatz am Trochanter minor auf, sind jedoch insgesamt sehr selten.

Disziplin: Krafttraining mit Gewichten und Kniebeugen, Sit-ups, Rudern, Bergauflaufen, Hürdenlauf, Hochsprung, Weitsprung, intensives Schusstraining beim Fußball.

Symptome: Schmerzen im Bereich der Leiste.

Bei Ruptur: plötzlicher stechender Leistenschmerz, reproduzierbar bei Hüftbeugung. Druckschmerz und Schwellung am Sehnenansatz.

Diagnostik: Druckschmerz am Trochanter minor (bei muskulösen Sportlern schwierig zu tasten), Schmerzauslösung bei Beugung des Hüftgelenkes gegen Widerstand, muskuläre Schwäche bei vollständiger Ruptur (Abb. 8).

Röntgen: Beckenübersicht zum Ausschluss eines knöchernen Ausrisses vor allem bei Heranwachsenden.

Therapie: konservativ: körperliche Schonung. Nach der Akutphase lokale Wärme. Antiphlogistika. Ggf. Steroidinjektion in den Muskelansatz mit nachfolgender 1-2-wöchiger Schonung und Vermeidung von Schnellkraftbelastung. Sonst wie bei Verletzung der Adduktoren.

Operation: bei vollständiger Ruptur.

Verletzungen der Bauchwandmuskulatur

Bei Rupturen oder Überlastungsverletzungen ist am häufigsten der $\mathrm{M}$. rectus abdominis geschädigt, selten der M. obliquus oder der $\mathrm{M}$. transversus abdominis. Der M. rectus abdominis entspringt am Sternum sowie 5.-7. Rippenknorpel und setzt am oberen Teil des Schambeines an der Symphyse an. Verletzungen sind normalerweise an der Ansatzstelle lokalisiert (Abb.9).

Ursache: Reizung, chronische Überlastung, Teilrupturen. Durch muskuläre Dysbalance vor allem bei exzentrischer Belastung und unzureichender Fähigkeit zur Stabilisierung (Rückschlagspiele). Akute Belastung beim Krafttraining, Sit-ups, Schusstraining beim Fußball, Aufschlagtraining. 


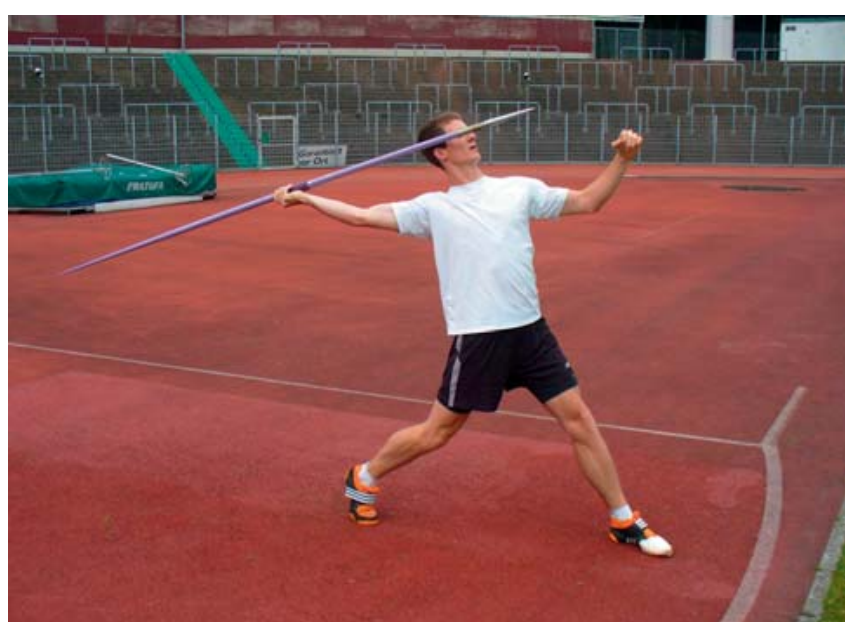

Abb. 10 Exzentrische Belastung der Bauchwandmuskulatur beim Speerwurf.

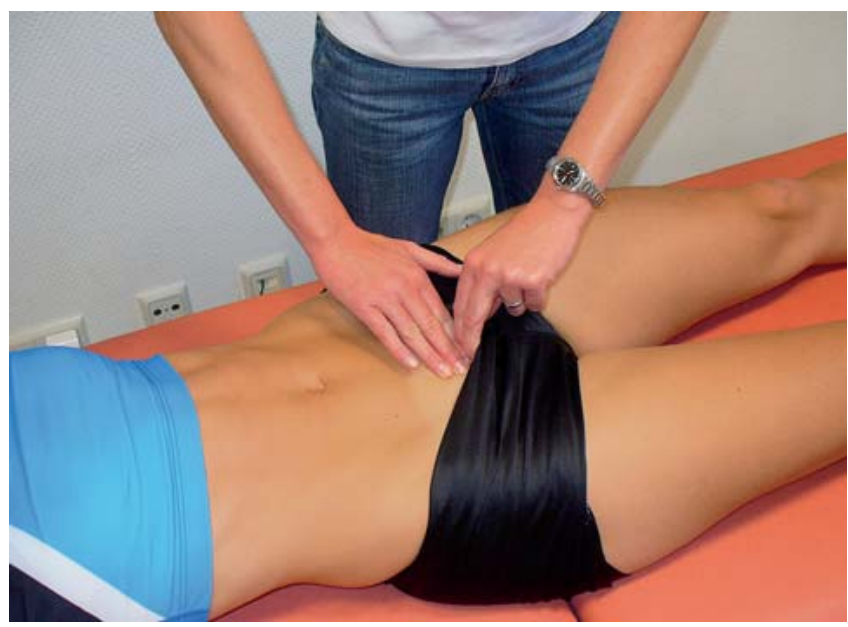

Abb. 11 Schmerzauslösung bei Druck über der Ansatzstelle des $M$. rectus abdominis am Os pubis.

Disziplin: Tennis, Badminton, Squash, Gewichtheben, Wurfdisziplinen (Speer), Rudern, Turnen, Hochsprung, Ringen (Abb.10).

Symptome: Schmerzen oberhalb der Symphyse, Schmerzen bei Einsatz der Bauchmuskulatur, bei kraftvoller Vorwärtsbewegung (Sprint).

Diagnostik: lokaler Druckschmerz am Os pubis, Schmerzauslösung bei Anheben der Beine gegen Widerstand in flacher Rückenlage, bei Verletzung/Ruptur der schrägen Bauchmuskulatur evtl. schwierig von einer Entzündung der inneren Organe (Appendizitis) zu unterscheiden, typischerweise ist jedoch die Schmerzsymptomatik bei Kontraktion der Muskulatur stärker ausgeprägt als bei Entspannung.

Bei Beschwerdepersistenz und v.a. Ruptur ggf. Sonografie und MRT (Abb.11).
Therapie: konservativ: körperliche Schonung. Antiphlogistika. Im Verlauf lokale Wärme. Ggf. lokale Steroidinjektion mit anschließender 2-wöchiger Schonung und Vermeidung von Schnellkraftbelastung. Aufnahme von Training und Wettkampf erst nach Abschluss des Heilungsprozesses, da sonst neue Rupturen oder Bauchwandhernien entstehen können.

\section{Operation bei anhaltenden Schmerzen.}

Verletzung des proximalen

$M$. rectus femoris

Der M. rectus femoris entspringt an der Spina iliaca anterior inferior und setzt mit der Quadrizepssehne an der Patella an, welche als Hypomochlion wirkt, und im weiteren Verlauf mit der Patellasehne an der Tuberositas tibiae. Es handelt sich daher um einen 2-gelenkigen Muskel, der als Hüftbeuger und Kniestrecker fungiert (Abb.12).

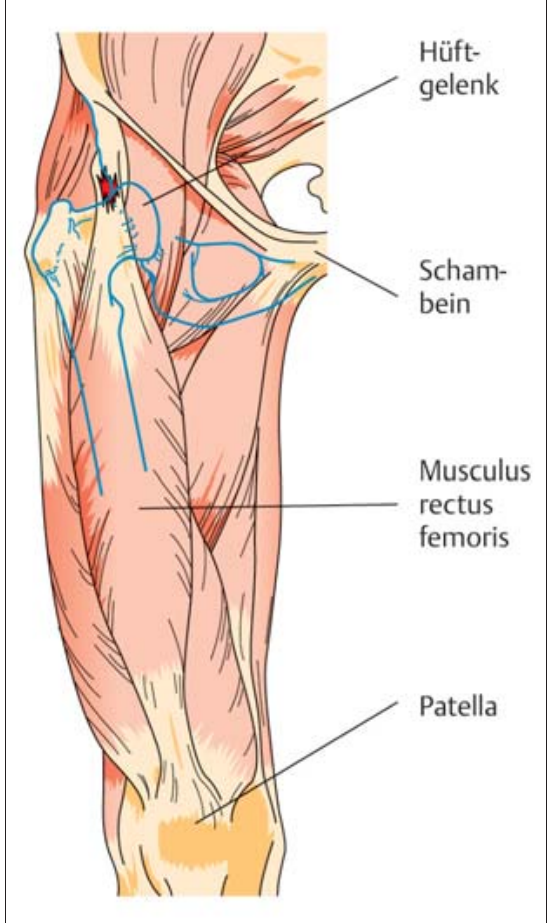

Abb. 12 Verletzung des M. rectus femoris im Ursprung der Muskel-Sehnen-Einheit.

Ursache: Überlastung, Teilruptur oder Ruptur im Ursprung oder oberen Drittel des Muskel-Sehnen-Überganges. Ausgelöst durch intensives Schusstraining beim Fußball, schnelle Antrittbewegungen wie beim Sprint, Krafttraining.

\section{Disziplin: Fußball, Sprint (Abb.13).}

Symptome: Schmerzen im Bereich der Leiste, lokaler Druckschmerz über dem Muskelursprung. Treppensteigen schmerzhaft.

Diagnostik: Bei vollständiger Ruptur kann der Muskel nicht mehr angespannt werden, Lücke palpabel. Schmerzauslösung bei Hüftbeugung oder Kniestreckung gegen Widerstand.

Konventionelles Röntgen, insbesondere bei Heranwachsenden zum Ausschluss einer Abrissfraktur (Abb.14).

Therapie: konservativ: wie bei Adduktorenverletzung.

Operation: bei vollständiger Ruptur und bei knöchernem Ausriss. 


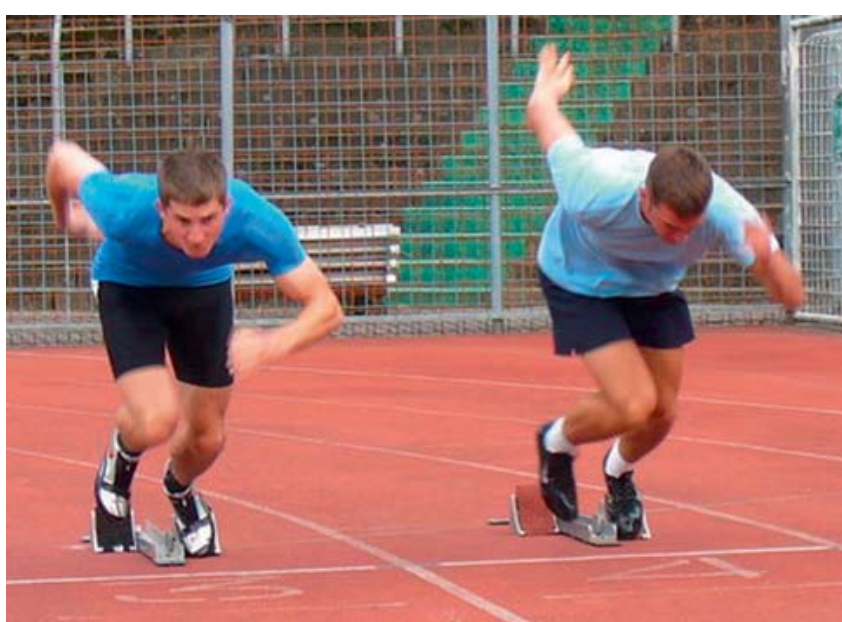

Abb. 13 Verletzungen des $\mathrm{M}$. rectus femoris treten häufig bei Sprintern auf.

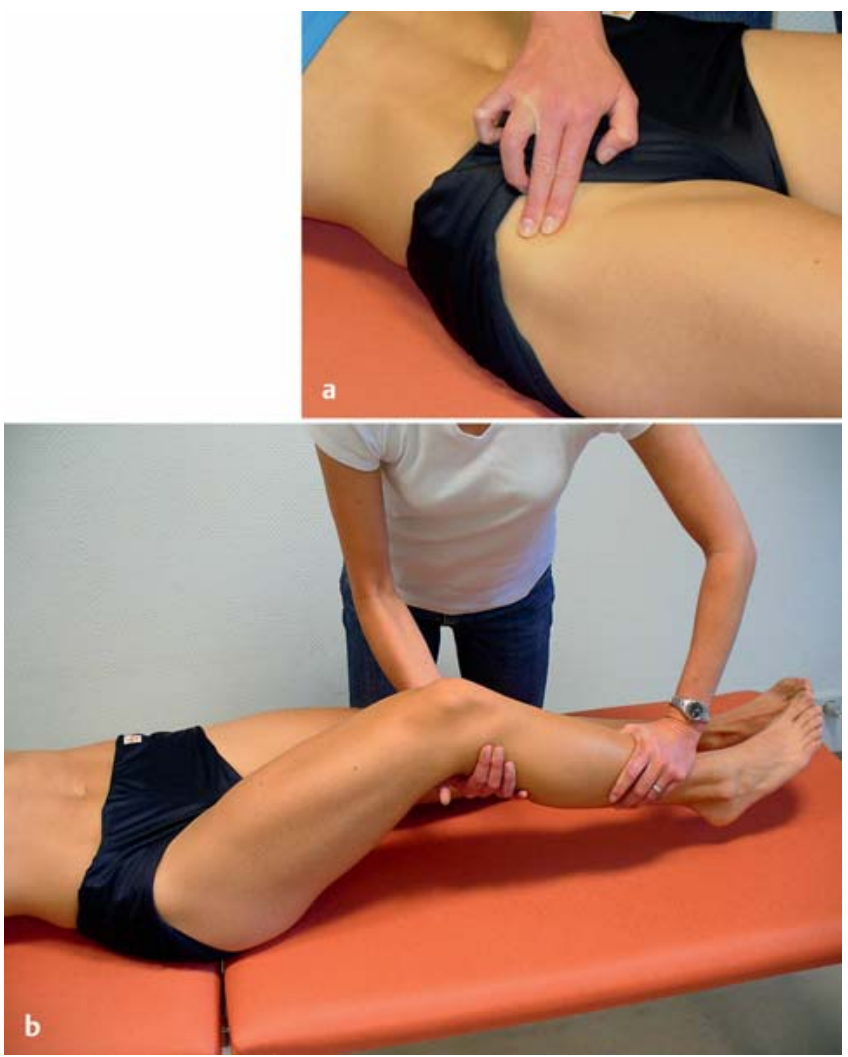

Abb. 14a und $b$ a Lokaler Druckschmerz im Bereich des Muskelursprunges. b Schmerz bei Streckung des Kniegelenkes gegen Widerstand.

Diagnostik: Anamnese: Gangbild des Patienten beobachten. Mit den Händen jeweils auf dem Trochanter major aufliegend hinter dem Patienten hergehen. Dabei lässt sich das Schnappen spüren. Ggf. lokaler Druckschmerz über Trochanter major oder Ansatz des M. iliopsoas.

Disziplin: Sprint- und Sprungdisziplinen.

Therapie: Bei alleinigem „Schnappen“ ist keine weitere Therapie erforderlich. Dehnungstherapie des Tractus iliotibialis und M. iliopsoas. Bei Schmerzen besteht die Indikation zur Operation.

Tractus iliotibialis: Traktopexie, Z-Plastik (Längsschnitt über Trochanter major, Faszienspaltung in Längsrichtung ca. $10-15 \mathrm{~cm}$, Schrägschnitt am proximalen Ende nach dorsal kaudal, ebenso am distalen Ende Schrägschnitt nach ventral kranial. Präparation der Faszienenden und Naht der Z-Plastik).

Iliopsoassehne: ggf. Einkerbung/Verlängerung der Iliopsoassehne.

Überlastung des Tractus iliotibialis

Die Glutealmuskeln setzen am Trochanter major an. Eine entzündliche Reizung kann zu Schmerzen in diesem Bereich führen.

Ursache: direktes Trauma, Achsfehlstellungen (Varusfehlstellung), Joggen auf einer Straßenseite.

Symptome: Schmerzen im Bereich des proximalen lateralen Femurs, Druckschmerz über Trochanter major.

Diagnostik: lokaler Druckschmerz, Schmerzen bei Abduktion des Beines gegen Widerstand, ebenso Schmerzen bei Dehnung, ggf. Kalzifikationen im konventionellen Röntgen.

\section{Coxa saltans/schnappende Hüfte}

Das Syndrom der „schnappenden Hüfte“ kann sowohl durch intraartikuläre Verletzungen des Hüftgelenkes selbst als auch der umgebenden Weichteile hervorgerufen werden. Die häufigsten Ursachen sind:

- Laterales Schnappen: Der verdickte Tractus iliotibialis gleitet über den Trochanter major oder eine verdickte Bursa.
- Vorderes tiefes Schnappen: Die Iliopsoassehne gleitet über die Eminentia iliopectinea oder durch eine entzündete bzw. verdickte Bursa iliopectinea.

- Intraartikuläre Pathologie: freie Gelenkkörper, Labrumriss etc.

Symptome: Der Sportler verspürt ein Schnappen in der Leiste oder am lateralen Oberschenkel. Manchmal ist dieses mit Schmerzen verbunden. Die Inzidenz ist bei Frauen höher.
Therapie: konservativ: Antiphlogistika, lokale Wärme, Schonung und Sportpause, Querfriktionsmassage. Bei längerer Reizung kann es zur Bursitis trochanterica kommen.

\section{Bursitiden (Schleimbeutelreizungen)}

Weichteilstrukturen wie Sehnen und Muskeln werden durch Schleimbeutel vor Reibung an knöchernen Strukturen geschützt. Man unterscheidet tiefe und 


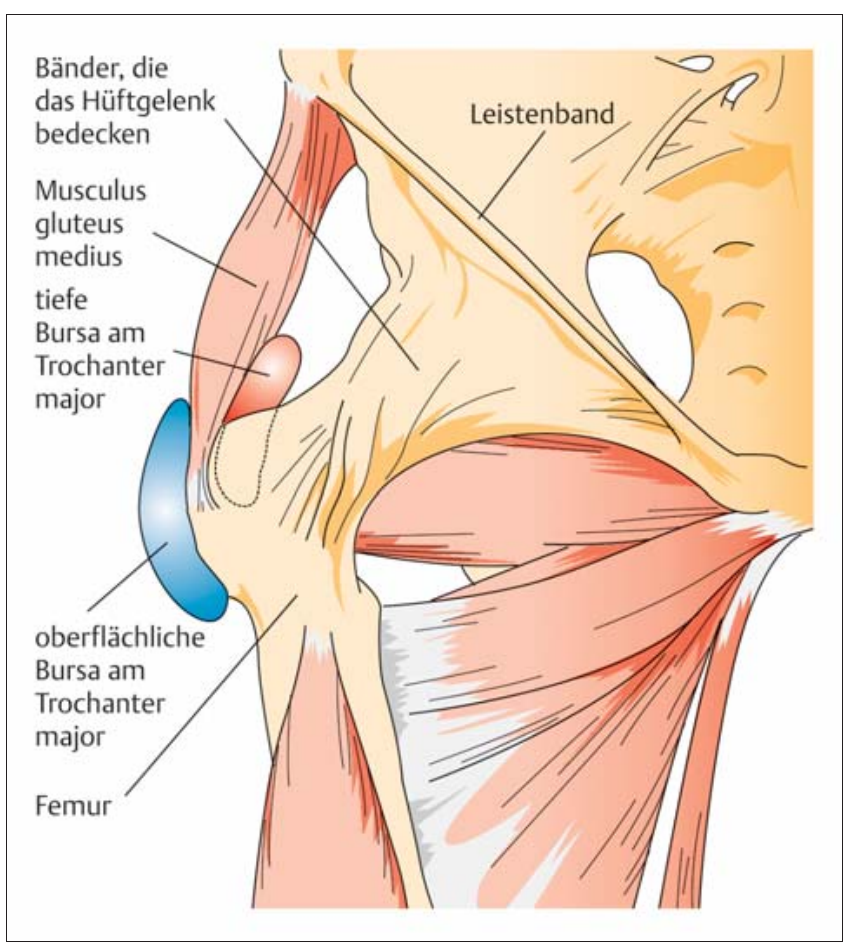

Abb. 15 Entzündung der oberflächlichen und tiefen Schleimbeutel am Trochanter major.
Druck bei Hypertrophie des M. sartorius oder zu enge Kleidung (Jeans) hervorgerufen werden.

Symptome: Schmerzen oder Dysästhesien an der Oberschenkelvorder- und außenseite.

Disziplin: Bodybilder, Gymnastik.

Diagnostik: Taubheit oder Überempfindlichkeit im Innervationsgebiet des Nervs, lokaler Druckschmerz an der Durchtrittsstelle des N. cutaneus femoris lateralis ca. $2 \mathrm{~cm}$ medial der Spina iliaca anterior superior. Messung der Nervenleitgeschwindigkeit. Diagnostische Lokalanästhetikainjektion um den Nerv.

Therapie: zunächst konservativ. Lokale Injektion mit Kombination von Lokalanästhetika und Kortikosteroid am Kompressionspunkt.

Operation: bei Beschwerdepersistenz chirurgische Dekompression des Nervs, ggf. Neurolyse.

\section{M.-piriformis-Syndrom}

Ursache: Muskuläre Dysbalancen, Verkürzung des M. piriformis und Beinlängendifferenz führen zur Reizung und Kompression des N. ischiadicus.

Symptome: ischialgieforme Schmerzen in der Wade, Schmerzen bei Dehnung des M. piriformis, Leistenbeschwerden, Schmerzen beim Sitzen und Bewegungen mit Hüftbeugung.

Disziplin: Laufdisziplinen, besonders Sprint und Hürdenlauf. len, Antiphlogistika, bei Hämatom oder Flüssigkeitsansammlung Punktion, ggf. Injektion mit Lokalanästhetikum und Kortikosteroid.

Operation: bei therapieresistenter Bursitis, freien Körpern oder Adhäsionen Indikation zur Bursektomie.

\section{Nervenkompressionssyndrome}

(„Nerveneinklemmungen“)

Meralgia paraesthetica

(,Jeanssyndrom“) Laufen, welche zum Schonhinken führen, nächtliche ausstrahlende Schmerzen in den Oberschenkel, Schmerzen bei Abduktion.
Diagnostik: lokaler Druckschmerz über dem M. piriformis, gut palpabler hypertoner Muskel in der Gesäßmuskulatur, Schmerzen bei Hüftbeugung und Innenrotation, Schmerzen bei kraftvoller Extension und Innenrotation des Oberschenkels durch den Untersucher (PaceZeichen).

Therapie: zunächst konservativ: Dehnungstherapie, bei der es zu ischialgieformen Schmerzen kommen kann, Kräftigung der übrigen Glutealmuskulatur, Antiphlogistika, ggf. Steroidinjektion (Cave: N. ischiadicus!).

Operation: Teilung der Piriformismuskulatur.
Ursache: Kompression des N. cutaneus femoris lateralis unter dem Ligamentum inguinale. Dies kann durch ein direktes Trauma, chronische Reizung durch 


\section{Komplikationen}

\section{Nach knöchernen Verletzungen}

Nach Abriss- oder Stressfrakturen kann es zu einer überschießenden Kallusbildung mit späterer Ossifikation kommen. Diese führen einerseits zu lokalen Schmerzen und Druckgefühl im Weichteilgewebe, andererseits kann es zur chronischen Reizung und Überlastung von Muskeln und Sehnen bis hin zur Ruptur oder Ausbildung von Bursitiden kommen.

\section{Nach Weichteilverletzungen}

Myogelosen

Im Muskel tastbare Knotenbildungen, die sich im Gegensatz zum Muskelhartspann auch in Narkose nicht entspannen lassen. Es handelt sich dabei um eine histologisch nachgewiesene hyaline Degeneration. Therapeutisch steht neben Massage und Elektrotherapie auch die Injektion eines lang anhaltenden Lokalanästhetikums zur Verfügung. Wichtiger ist jedoch die Prävention: Aufwärmund Auslauftraining sowie Dehnung nach der Belastung.

\section{Myositis ossificans}

Dabei handelt es sich um eine heterotope Knochenbildung in der Muskulatur, die posttraumatisch durch Kalzifikation und Verknöcherung eines Hämatoms entsteht. Ein solches intramuskuläres
Hämatom tritt vor allem nach Prellungen bei Kontaktsportarten wie Fußball, Rugby, Handball und Eishockey auf, aber auch nach Teilrupturen von Muskeln, die in allen Disziplinen auftreten können. Oberste Priorität hat daher die Prävention durch Blutstillung und Begrenzung des Hämatoms unmittelbar nach Entstehung der Verletzung: Entlastung des Muskels, Kryotherapie und Kompression! Eine unsachgemäße Behandlung mit zu früh einsetzender lokaler Wärmetherapie, harten Massagen und Dehnungsübungen über die Schmerzgrenze hinaus fördern die Entstehung einer solchen Myositis ossificans. Therapeutisch haben Röntgenreizbestrahlungen gute Erfolge, sind jedoch mit einer hohen Strahlenbelastung verbunden. Medikamentös kann bei fehlenden Kontraindikationen Indometacin täglich $(3 \times 25 \mathrm{mg}$ bis $3 \times 50 \mathrm{mg}$ ) verordnet werden. Bei Einschränkungen von Muskelkraft oder Bewegungsausmaß ist die operative Therapie indiziert, bei der die Ossifikation vollständig entfernt wird.

\section{Literatur}

${ }^{1}$ Bachelier F, Steimer O, Kohn D, Dienst M. Arthroskopie des Hüftgelenkes. OP-JOURNAL 2007; 23: 22-28

2 Brukner P, Bennell K. Stress fractures in female athletes. Diagnosis, management and rehabilitation. Sports Med 1997; 24: 419 429

${ }^{3}$ Hopp S. Osteitis pubis. Deutsche Zeitschrift für Sportmedizin 2008; 59: 100 - 101

${ }^{4}$ Lohrer H, Nauck T. [Proximal adductor longus tendon tear in high level athletes. A report of three cases]. Sportverletz Sportschaden 2007; 21: 190-194
${ }^{5}$ Lynch SA, Renstrom PA. Groin injuries in sport: treatment strategies. Sports Med 1999; 28: $137-144$

${ }^{6}$ Major NM, Helms CA. Pelvic stress injuries: the relationship between osteitis pubis (symphysis pubis stress injury) and sacroiliac abnormalities in athletes. Skeletal Radiol 1997; 26: 711 - 717

7 Miller MH, Howard RF, Plancher KD. Operationsatlas Sportorthopädie - Sporttraumatologie. München: Elsevier; 2004

8 Pavlov H, Nelson TL et al. Stress fractures of the pubic ramus. A report of twelve cases. J Bone Joint Surg [Am] 1982; 64: 1020-1025

9 Peterson L, Renström P. Verletzungen im Sport. Köln: Deutscher Ärzte Verlag; 2002

${ }^{10}$ Rossi F, Dragoni S. Acute avulsion fractures of the pelvis in adolescent competitive athletes: prevalence, location and sports distribution of 203 cases collected. Skeletal Radiol 2001; 30: 127-131

11 Thorne DA, Datz FL. Pelvic stress fracture in female runners. Clin Nucl Med 1986; 11: $828-829$

12 Tscherne H, Pohlemann T. Tscherne Unfallchirurgie, Becken und Acetabulum. 1. Aufl. Berlin: Springer; 1998

\section{Dr. med. Julia Heinzmann Assistenzärztin}

Prof. Dr. med. Tim Pohlemann Klinikdirektor

Klinik für Unfall-, Hand- und Wiederherstellungschirurgie Universitätsklinikum des Saarlandes Kirrberger Straße 66421 Homburg 